\title{
The Jets of NGC 6543
}

\author{
B.P. Francis, M. Bryce, M.F. Graham, A.J. Holloway
}

Jodrell Bank Observatory, Department of Physics and Astronomy, University of Manchester, Macclesfield, Cheshire SK119DL, UK

\section{J.A. López}

Instituto de Astronomía, UNAM, Km 103 Carretera Tijuana-Ensenada, 22860 Ensenada, B. C., Mexico

\begin{abstract}
High spectral resolution observations of the high speed jets of NGC 6543 are presented. When deprojected, these give velocities of $V_{\text {north }}=-82.0 \mathrm{~km} \mathrm{~s}^{-1}$ and $V_{\text {south }}=63.8 \mathrm{~km} \mathrm{~s}^{-1}$.
\end{abstract}

\section{Observations and results}

The Planetary Nebula NGC 6543 has several long narrow jet-like features visible in $[\mathrm{NII}]$ which are thought to be evidence of precessing jets (Harrington \& Borkowski 1994). The two brightest jets were observed with the Manchester echelle spectrometer (Meaburn et al. 1984) combined with the f/7.9 2.1m San Pedro Martir telescope on 17/18 May 2001. The spatial resolution was 0.35 arcsec pixel ${ }^{-1}$ on the SITE $31024 \times 1024\left(24 \times 24 \mu \mathrm{m}^{2}\right)$ pixel CCD detector. A slit width of $70 \mu \mathrm{m}(0.87 \mathrm{arcsec})$ was used, giving a velocity resolution of 4.5 $\mathrm{km} \mathrm{s}^{-1}$. The slit positions are shown in Fig. 1. Observaton times were 1800 seconds for both slit positions.

Fig. 2(a) shows the [NII] $6584 \AA$ nebular emission line for slit position 1 . The jet is visible at around $V_{H E L}=-100 \mathrm{~km} \mathrm{~s}^{-1}$, giving a radial velocity with respect to the systemic, $V_{r}=-41.0 \mathrm{~km} \mathrm{~s}^{-1}$. Slit postion 2 (Fig. 2(b)) shows the jet along with a feature at a smaller red shift, probably emission from the polar cap. The jet has $V_{r}=31.9 \mathrm{~km} \mathrm{~s}^{-1}$. If the observed ellipse A (Fig. 1) is actually a circular ring, an inclination of its axis to the plane of the sky of $\sim 30^{\circ}$ is implied. Therefore the deprojected jet speed is $V_{r} / \sin 30^{\circ}=2 V_{r}$, giving $V_{\text {north }}=-82.0 \mathrm{~km} \mathrm{~s}^{-1}$ and $V_{\text {south }}=63.8 \mathrm{~km} \mathrm{~s}^{-1}$ in the north and south jets respectively. An interesting feature in Fig. 3 is the faint blue shifted component which is possibly a remnant from a time when the southern precessing jet was angled towards us as opposed to away from us as it is now.

\section{References}

Meaburn, J. et al. 1984, MNRAS, 210, 463

Harrington, J. P. \& Borkowski, K. J. 1994, BAAS, 26, 1469 


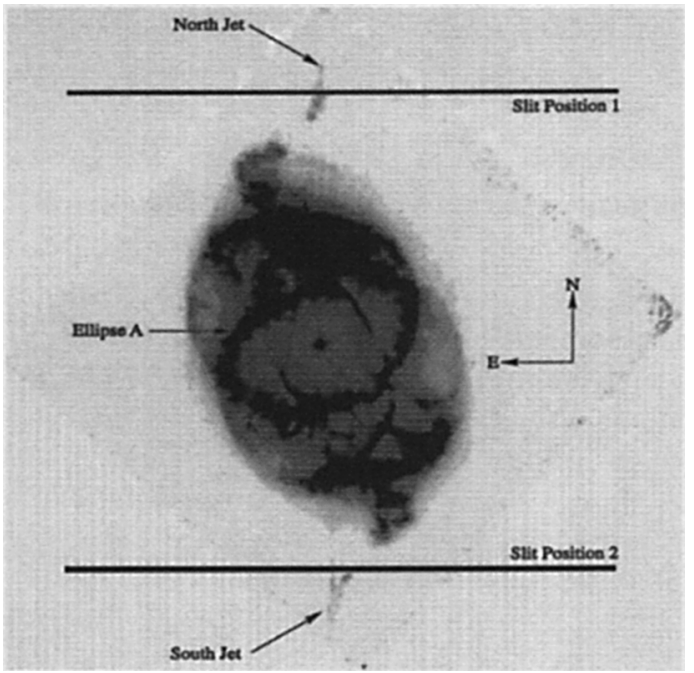

Figure 1. The 36x36 arcsecond WFPC2 image (ref. GO 5403, PI: J.P. Harrington) showing slit positions 1 and 2 .
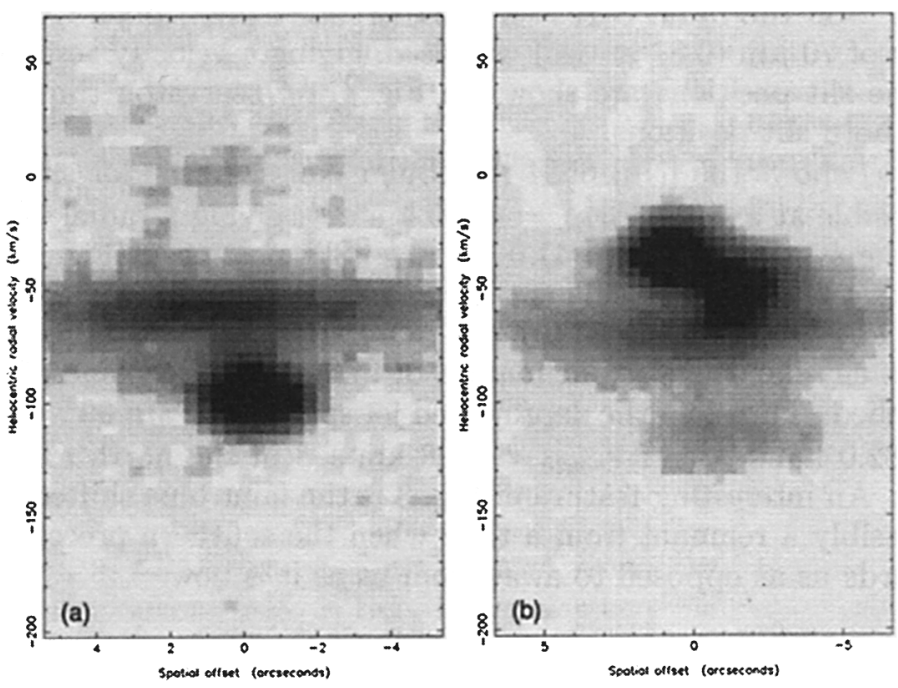

Figure 2. [NII] $6584 \AA$ emission lines from slit position 1 (a) and slit position 2 (b). East is a positive spatial offset. 